\title{
A Newborn Presenting With Bilious Vomiting: A Case Report of A Jejunal Atresia
}

\author{
Joo Y Cho ${ }^{1 *}$ and Valerie Plant ${ }^{2}$ \\ ${ }^{1}$ Department of Radiology, Children's Hospital of Richmond, USA \\ ${ }^{2}$ Department of Surgery, Virginia Commonwealth University Medical Center, USA \\ *Corresponding author: Joo Y. Cho, MD, Department of Radiology, Children's Hospital of Richmond, 1200 East Broad Street, West Hospital, Richmond, \\ Virginia 23298-0615, USA
}

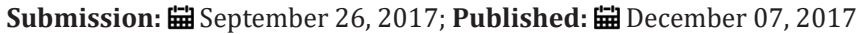

\begin{abstract}
Jejunal atresia is a relatively rare cause of proximal small bowel obstruction with incidence of 1-3 per 10,000 live births. The condition is caused by in utero vascular insult, and it occurs more commonly in the proximal jejunum. These patients present with abdominal distention and bilious emesis. Workup usually starts with an abdominal radiograph, which will show dilated stomach and proximal bowel loops. An UGI is performed to exclude midgut volvulus and determine a more precise location of obstruction. Once the diagnosis is made, definitive treatment is surgical resection of the atretic segment and primary anastomosis. Malrotation with midgut volvulus is an important differential diagnosis to exclude, which is a surgical emergency.
\end{abstract}

Keywords: Jejunal atresia; Duodenojejunal junction; Midgut volvulus; In utero vascular insult

Abbreviations: UGI: Upper Gastrointestinal; DJJ: Duodenojejunal Junction; APGAR: Appearance, Pulse, Grimace, Activity, and Respiration

\section{Introduction}

Jejunal atresia is one of several causes of proximal small bowel obstruction. Incidence is about 1-3 per 10,000 live births [1], and thought to be caused by in utero vascular insult [2]. Approximately $2 / 3$ of jejunal atresia occur in the proximal segment, and atresia is much more common than jejunal stenosis [1]. Jejunal atresia has been shown to be associated with abdominal wall defects, cystic fibrosis, and meconium ileus [3]. There is no gender predilection. Patients with proximal jejunal obstruction usually present with bilious emesis, necessitating prompt work up and surgical intervention when necessary. Although classic abdominal radiographic findings may be sufficient to make the diagnosis, the appearance can often overlaps with other causes of proximal bowel obstruction. An upper gastrointestinal (UGI) study can beperformed to determine the level of obstruction more precisely. The current case reviews the presentation, imaging work-up, surgical intervention, and post-operative management of a patient born with jejunal atresia.

\section{Case Presentation}

An infant girl was born at 35 weeks gestation to a G2P3 mother via Caesarean section for non-reassuring fetal heart tracings, decreased fetal movement, and oligohydramnios. APGAR scores were 9 and 9. It was unclear if mother had regular prenatal care, but a prenatal ultrasound was concerning for intestinal obstruction. The patient was born at a community hospital with abdominal distention and had an episode of bilious emesis. Abdominal radiograph showed abnormal gas-dilated stomach, duodenum, and proximal jejunum, collectively known as the "triple bubble" sign (Figure 1) [4]. There was no bowel gas in the distal bowel loops. The patient was urgently transferred to the children's hospital for further care.

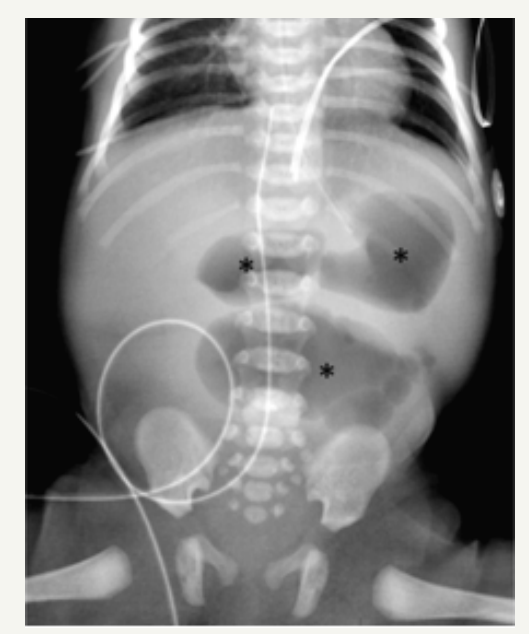

Figure 1: AP supine abdominal radiograph of a newborn infant with abdominal distension shows gaseous distention of the stomach, duodenum, and the proximal jejunum, collectively known as the "triple bubble sign" $(*)$. No bowel gas is seen distally. 
Upon arrival, an UGI study was performed as part of diagnostic work-up. Normal position of the duodenal jejunal junction (DJJ) was confirmed, and the level of obstruction appeared to be several centimeters distal from the ligament of Treitz (Figure 2). There was complete obstruction in the proximal jejunum with contrast material opacifying the dilated stomach, duodenum, and proximal jejunum. The appearance of the obstruction did not resemble a midgut volvulus. A nasogastric tube was in place to decompress the stomach and bowel proximal to the obstruction.

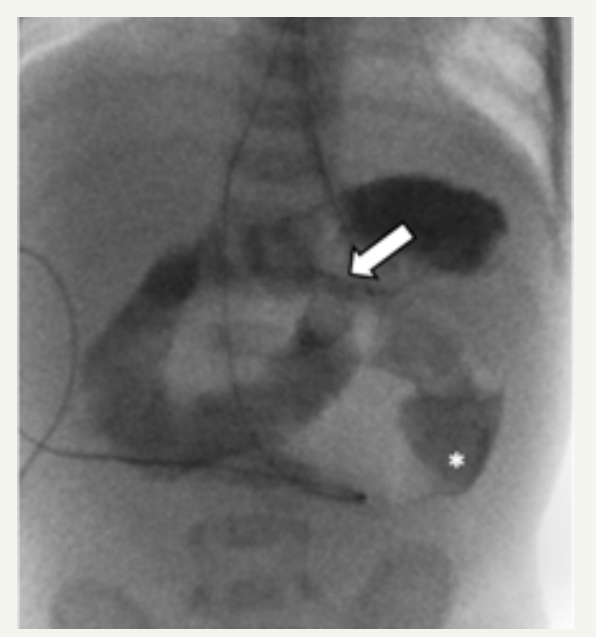

Figure 2: AP fluoroscopic view of an upper gastrointestinal study (UGI) shows normal position of the duodenojejunal junction (DJJ) and dilated proximal jejunum. Obstruction was several centimeters distal from the ligament of Treitz $(*)$.

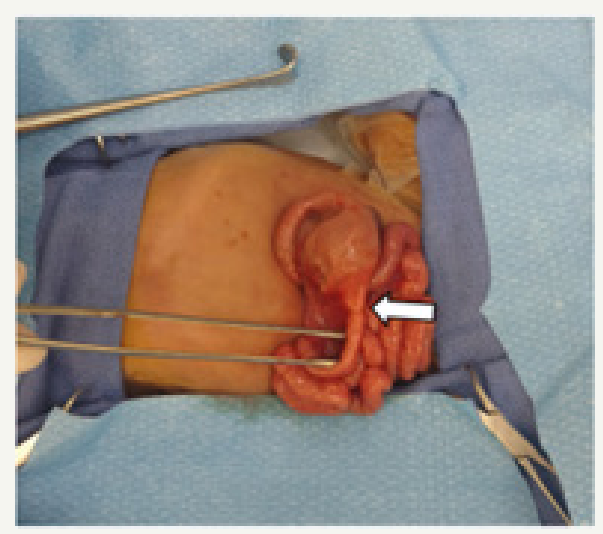

Figure 3: Surgical pathology. The proximal jejunum is markedly dilated with focal area of narrowing, consistent with the site of atresia, which was approximately $5 \mathrm{~cm}$ distal to the ligament of Treitz.

The infant was admitted to the neonatal intensive care unit, and surgery was performed the next day. A transverse incision was made in the right upper quadrant. The small bowel was eviscerated and closely inspected from ligament of Treitz to rectum. Proximal jejunum was moderately dilated. There was an abrupt diameter change at the area of atresia, which was approximately five $\mathrm{cm}$ distal to the ligament of Treitz (Figure 3). After division of the mesentery, a segment measuring three $\mathrm{cm}$ of jejunum proximal to the atresia and one $\mathrm{cm}$ distal to the atresia was resected. Injection of saline into the distal bowel confirmed patency of the remaining small and large bowel loops. A single layer anastomosis was created with absorbable suture in an interrupted fashion. The mesentery defect was also closed to prevent internal hernia.

Our patient had prolonged post-op functional ileus, with no return of bowel function for several days. Her nasogastric tube continued to have bilious output, with volumes up to $28 \mathrm{ml} / \mathrm{kg} /$ day. Her abdomen remained non-distended, and abdominal imaging revealed air throughout her bowels. Once her nasogastric tube output decreased to about $10 \mathrm{ml} / \mathrm{kg} /$ day, trickle feeds were initiated via the tube. She tolerated trickle feeds well and had low residual volumes. She finally had bowel function on post-op day 16 . Her feeds were advanced, and she continued to have bowel function. She was advanced to bolus feeds by mouth and was progressing well. She was discharged home three weeks post-op.

\section{Discussion}

While obstruction in the duodenum is thought to be due to failure of re-canalization, jejunal or ileal atresia are thought to be secondary to in utero vascular insults [1-3].

There are several types of jejunal atresia. Type I has an obstructing membrane without disruption of the serosa or mesentery. Type II atresia is characterized by a fibrous band between the two (proximal and distal) atretic ends. Type IIIa has two blind intestinal segments and a mesenteric defect. Type IIIb has a dilated, blind-ending proximal pouch and the distal blind-ending segment wrapped around the ileocolic artery. Type IV atresia is characterized by multiple segments of atresias [1,3]. Our patient had a type I jejunal atresia.

Jejunal atresia can occur anywhere but more commonly in the proximal segment [1]. Maternal polyhydramnios is present in up to $24 \%$ of patients [3], and prenatal imaging will show dilated proximal bowel loops. Soon after birth, patients with proximal jejunal atresia will present with bilious emesis [5], while patients with a more distal jejunal atresia will present with jaundice and delayed clinical onset of signs [3].

One important diagnosis to exclude in neonates presenting with bilious emesis is malrotation with midgut volvulus, which results from twisting of mesentery around the SMA axis, leading to bowel obstruction and ischemia [6]. In this condition, timely surgical intervention is necessary to prevent bowel infarction. Although abdominal radiograph can demonstrate proximal bowel dilation, the appearance is non-specific and it can be difficult to distinguish between jejunal atresia and midgut volvulus. Abdominal radiographs can also be completely normal in these conditions. An UGI is performed not only to determine the level of obstruction, but to try to diagnose or exclude a midgut volvulus. Efforts should be made to determine the position of the DJJ, which is abnormally positioned in patients with malrotation. Unlike midgut volvulus, jejunal atresia is not a surgical emergency, and careful planning may take place before the patient is brought to the operating room. 
Definitive treatment is surgical resection of the atretic segment with primary anastomosis.

In conclusion, jejunal atresia mostly occurs in the proximal jejunum, and these patients present with bilious emesis. Abdominal radiographs are suggestive of the diagnosis, and UGI can help determine the level of obstruction and also to exclude a midgut volvulus, which is a surgical emergency. Definitive treatment is surgery.

\section{References}

1. Coley BD (2013) Caffey's Pediatric Diagnostic Imaging. (12 ${ }^{\text {th }}$ edn), Elsevier, Philadelphia, USA.
2. Walters M, Robertson R (2016) Pediatric Radiology: The Requisites. (4 $4^{\text {th }}$ edn), Elsevier Health Sciences, Philadelphia, USA

3. Wyllie R, Hyams JS, Kay M (2016) Pediatric Gastrointestinal and Liver Disease.

4. Mandell J (2013) Core Radiology: A Visual Approach to Diagnostic Imaging. Cambridge University Press, New York, USA.

5. Huisman T (2011) Pediatric Imaging: Case Review. Elsevier, Philadelphia, USA.

6. Cho JY, Lillehei C, Callahan MJ (2017) Practical Approach to Imaging of the Pediatric Acute Abdomen. Current Treatment Options in Pediatrics 3(3): 175-192. 